\title{
ON A CRITERION FOR THE UNIVALENCE OF HOLOMORPHIC FUNCTIONS
}

\section{KEE-WAI LAU AND MING-CHIT LIU}

ABSTRACT. A sufficient condition for the univalence of holomorphic functions in the unit disc is given in terms of $\left|f^{\prime \prime} / f^{\prime}\right|$.

Let $f$ be a function nonconstant and holomorphic in $D=\{z \in \mathbf{C}:|z|<1\}$. Let $\alpha>0$ and let

$$
\|f\|_{\alpha}=\sup _{z \in D}\left(1-|z|^{2}\right)^{\alpha}\left|f^{\prime \prime}(z) / f^{\prime}(z)\right| .
$$

In this paper we shall prove

ThEOREM. If $\alpha \in(0,1]$ write

$$
K_{\alpha}=\left(B_{\alpha}^{2}+4(2-\alpha)\right)^{1 / 2}-B_{\alpha},
$$

where

$$
B_{\alpha}=Y_{\alpha}\left(1-Y_{\alpha}^{2}\right)^{\alpha+1} 2(\alpha+1)(2 \alpha+1)^{\alpha+3 / 2}(2 \alpha)^{-(1+\alpha)}
$$

and

$$
Y_{\alpha}=\left(\left\{\left(4 \alpha^{2}+6 \alpha+1\right) /(2 \alpha+1)\right\}^{1 / 2}-1\right) /(2 \alpha+2) .
$$

If $\|f\|_{\alpha}<K_{\alpha}$ then $f$ is univalent in $D$.

The proof of our theorem depends on the following lemma:

LEMMA W (KOROLLAR 1 in [5]). If

$$
E_{\alpha}=\left\{g: g \text { is holomorphic in } D \text { and }|g(z)|<K_{\alpha}\left(1-|z|^{2}\right)^{-\alpha} \text { in } D\right\} \text {, }
$$

then

$$
B_{\alpha}=\min \left\{\beta \in \mathbf{R}:\left|g^{\prime}(z)\right| \leqslant \beta K_{\alpha} /\left(1-|z|^{2}\right)^{1+\alpha} \text { in } D \text { for all } g \in E_{\alpha}\right\},
$$

where $B_{\alpha}$ and $K_{\alpha}$ are defined as in the Theorem.

Proof of The Theorem. We suppose

$$
\left(1-|z|^{2}\right)^{\alpha}\left|f^{\prime \prime} / f^{\prime}\right|<K_{\alpha}
$$

and $\alpha \in(0,1]$. Hence

$$
\left(1-|z|^{2}\right)^{1+\alpha}\left|f^{\prime \prime} / f^{\prime}\right|^{2}<K_{\alpha}^{2} .
$$

Putting $g=f^{\prime \prime} / f^{\prime}$ we apply Lemma W and obtain

Received by the editors August 10, 1979 and, in revised form, December 4, 1979.

1980 Mathematics Subject Classification. Primary 30C55. 


$$
\left(1-|z|^{2}\right)^{1+\alpha}\left|\left(f^{\prime \prime} / f^{\prime}\right)^{\prime}\right|<B_{\alpha} K_{\alpha}
$$

Therefore

$$
\left(1-|z|^{2}\right)^{1+\alpha}|\{f, z\}|<B_{\alpha} K_{\alpha}+\frac{1}{2} K_{\alpha}^{2}=2(2-\alpha),
$$

where $\{f, z\}=\left(f^{\prime \prime} / f^{\prime}\right)^{\prime}-\frac{1}{2}\left(f^{\prime \prime} / f^{\prime}\right)^{2}$ is the Schwarzian derivative. The last inequality follows directly from (1). Beesack $[2,(2.6)$ and $(2.8)]$ has proved: If $\lambda \in[1,2]$ and

$$
\left(1-|z|^{2}\right)^{\lambda}|\{f, z\}|<2(3-\lambda)
$$

then $f$ is univalent. Put $\lambda=1+\alpha$ and the Theorem follows.

REMARKs. Our attention is restricted to $K_{\alpha}>1$ because Becker [1, Korollar 4.1] proved that $f$ is univalent in $D$ if $\|f\|_{1}<1$. As for $\alpha<1,\|f\|_{\alpha}>\|f\|_{1}$ we have that $f$ is univalent in $D$ if $\|f\|_{\alpha}<1$.

Yamashita [6, Theorem] has proved that if $\alpha \in[0,1], \beta=2^{1+\alpha}$ and if

$$
\|f\|_{\alpha}<k_{\alpha}=\left(\beta^{2}+4(2-\alpha)\right)^{1 / 2}-\beta
$$

then $f$ is univalent in $D$. In particular $k_{0}=1.464 \ldots$ and $k_{\alpha}>1$ when $\alpha \in$ $[0,0.416 \ldots)$. By (4) we see that $k_{\alpha}$ is a strictly decreasing function of $\beta$. So comparing (1) with (4) we have $K_{\alpha}>k_{\alpha}$ everywhere on $(0,1]$ since $B_{\alpha}<2^{1+\alpha}$ on $(0,1]$, which can be seen with the help of a result due to Robertson [4, (2.9)], namely $B_{\alpha}<(1+\alpha)^{1+\alpha} \alpha^{-\alpha}$ if $\alpha>0$. In fact $\lim _{\alpha \rightarrow 0+} K_{\alpha}=2>1.464 \ldots=k_{0}$ and we can prove that $K_{\alpha}>1$ when $\alpha \in(0,0.575 \ldots) \supset(0,0.416 \ldots)$. Therefore except at $\alpha=0$ our result improves the theorem in [6] for both the values of $k_{\alpha}$ and the range of $\alpha$. Since the value of $B_{\alpha}$ (see (2)) in Lemma W is the minimum of all upper bounds $\beta$ and hence cannot be lowered anymore we expect that our theorem cannot be improved significantly if Beesack's result (3) is still adopted.

At $\alpha=0$, Kudryashov [3, Theorem 1] proved that $f$ is univalent in $D$ if $\|f\|_{0}<M=3.05 \ldots$ where $M$ is a root of the equation,

$$
8\left(x(x-2)^{3}\right)^{1 / 2}-3(4-x)^{2}=12 .
$$

The authors wish to thank the referee for his valuable suggestions which improve the presentation of this paper.

\section{REFERENCES}

1. J. Becker, Löwnersche Differentialgleichung und quasikonform fortsetzbare schlichte Funktionen, J. Reine Angew. Math. 255 (1972), 23-43.

2. P. R. Beesack, Nonoscillation and disconjugacy in the complex domain, Trans. Amer. Math. soc. 81 (1956), 211-242.

3. S. N. Kudryashov, On some criteria for the univalence of analytic functions, Math. Notes 13 (1973), 219-223.

4. M. S. Robertson, A distortion theorem for analytic functions, Proc. Amer. Math. Soc. 28 (1971), 551-556.

5. K. J. Wirths, Über holomorphe Funktionen, die einer Wachstumsbeschränkung unterliegen, Arch. Math. (Basel) 30 (1978), 606-612.

6. S. Yamashita, On a theorem of Duren, Shapiro and Shields, Proc. Amer. Math. Soc. 73 (1979), 180-182.

Department of MAThematics, University of Hong Kong, Hong Kong 